\title{
Facile Synthesis of Magnetic Covalent Organic Framework with Three-Dimensional Bouquet-Like Structure for Enhanced Extraction of Organic Targets
}

\author{
Sijing He, ${ }^{\dagger, \ddagger}$ Tao Zeng, ${ }^{\|}$Saihua Wang, ${ }^{\dagger, \ddagger}$ Hongyun Niu, ${ }^{\dagger}$ and Yaqi Cai ${ }^{*}, \dagger, \downarrow, \S$ \\ ${ }^{\dagger}$ State Key Laboratory of Environmental Chemistry and Ecotoxicology, Research Center for Eco-Environmental Sciences, Chinese \\ Academy of Sciences, Beijing 100085, China \\ ${ }^{\ddagger}$ University of Chinese Academy of Sciences, Beijing 100049, China \\ ${ }^{\S}$ Institute of Environment and Health, Jianghan University, Wuhan 430056, China \\ "College of Environment, Zhejiang University of Technology, Hangzhou 310032, China
}

Supporting Information

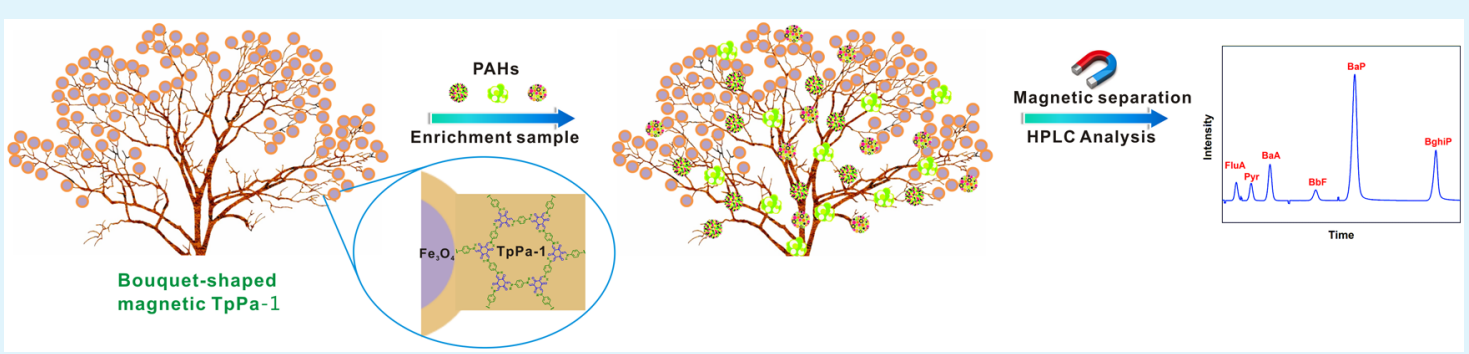

ABSTRACT: A facile strategy for the fabrication of novel bouquet-shaped magnetic porous nanocomposite via grafting a covalent organic framework (COF, TpPa-1) onto the surface-modified $\mathrm{Fe}_{3} \mathrm{O}_{4}$ nanoparticles $\left(\mathrm{Fe}_{3} \mathrm{O}_{4} \mathrm{NPs}\right)$ was reported. The magnetic TpPa-1 (a COF synthesized from 1,3,5-triformylphloroglucinol (Tp) and p-phenylenediamine (Pa-1)) contains clusters of core-shell magnetic nanoparticles and interconnected porous TpPa-1 nanofibers. Thus, it possesses larger specific surface area, higher porosity, and supermagnetism, making it an ideal sorbent for enrichment of trace analytes. Its performance was evaluated by the magnetic solid-phase extraction (MSPE) of trace polycyclic aromatic hydrocarbons (PAHs) from environmental samples prior to high-performance liquid chromatographic analysis. The results indicated that the magnetic TpPa1 possessed superior enrichment capacity of such organic compounds.

KEYWORDS: magnetic covalent organic framework, bouquet-like structure, solid-phase extraction, polycyclic aromatic hydrocarbons, environmental water samples

\section{INTRODUCTION}

As a novel pattern of solid-phase extraction (SPE), magnetic solid-phase extraction (MSPE) based on magnetic or magnetically modified sorbents, has been developed as a promising technique in preconcentration and separation. ${ }^{1}$ With respect to the MSPE procedure, the analytes can be rapidly adsorbed onto the magnetic sorbents in the sample solution and then be collected by means of applied magnetic field, which greatly simplifies the pretreatment procedure and enhances extraction efficiency. Hence, the development of a new type of efficient sorbent is the core of MSPE technique. So far, functionalized porous materials with distinct magnetic property have been successfully applied in MSPE, such as activated carbon, ${ }^{2,3}$ zeolitic imidazole frameworks (ZIFs), ${ }^{4,5}$ conjugated microporous polymers (CMPs), ${ }^{6}$ and metal-organic frameworks (MOFs). ${ }^{7-12}$

Generally, these magnetic porous materials present a coreshell architecture including a magnetite core and a porous layer. The core-shell nanostructure can impede the aggregation of magnetite cores and prevent loss of magnetism while allowing feasible surface modification, enabling it to be a desired candidate for MSPE. ${ }^{13}$ However, inherently low shell content of the core-shell sorbent limits its adsorption performance in MSPE. As we all know, the structure and properties of the shell material dramatically affect the adsorption performances of the magnetic porous nanocomposites. Therefore, choosing the suitable coating substances and controlling their shapes is an effective approach to further enhance extraction efficiency of the sorbent.

Covalent organic frameworks (COFs) are an emerging type of crystalline porous polymers ingeniously constructed with organic building blocks via robust covalent bonds. ${ }^{14,15}$ They are synthesized from lightweight elements $(\mathrm{H}, \mathrm{B}, \mathrm{C}, \mathrm{N}, \mathrm{O}$, and $\mathrm{Si}$ ) through the same protocols of reticular chemistry as MOFs. ${ }^{16}$

Received: October 26, 2016

Accepted: December 29, 2016

Published: January 11, 2017 
Scheme 1. Synthesis and Application of the Bouquet-Like Magnetic TpPa-1 Sorbent

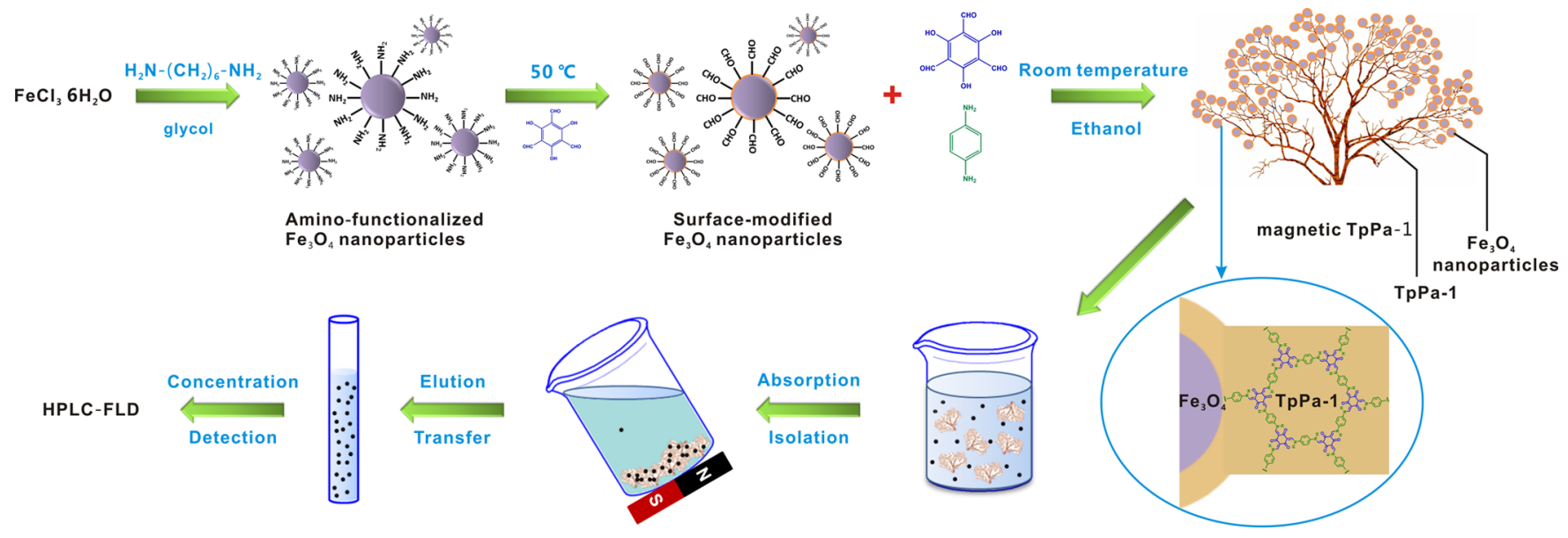

The fascinating features like permanent porosity, tunable pore size, large specific surface area, low density, and facilely tailored functionality render COFs superior potential in a wide variety of applications, such as gas storage, ${ }^{17-20}$ catalysis, $^{21-25}$ and optoelectronics. $^{26-29}$ Compared with their analogues, COFs overcome the problems of water and moisture instability (MOFs), ${ }^{30,31}$ the difficulty to regulate their structures and functionalities (ZIFs, activated carbon), or the lack of sustainable synthesis (CMPs). ${ }^{32,33}$ Among them, TpPa-1 was reported to have exceptional stability in aqueous, acidic, and basic medium for the proton tautomerism. ${ }^{34-39}$ Based on the in-built covalent bond architecture, one could anticipate that COFs might become more suitable scaffolds than other porous materials for decorating magnetic nanoparticles and therefore emerge as efficient and versatile MSPE sorbents.

Herein, inspired by the gypsophila bouquet, we designed and synthesized a novel bouquet-shaped magnetic $\mathrm{TpPa}-1$ with the combination of superiorities of core-shell configuration and COFs for the first time. The synthetic and MSPE procedures of magnetic TpPa-1 nanocomposites are shown in Scheme 1. With this simple and moderate methodology, core-shell magnetic "flowers" and porous "stems" are linked together via covalent bonding assembly, which allows for facile withdrawal of the obtained hybrid magnetic $\mathrm{TpPa}-1$ by magnetic decantation. Considering the adsorption sites were mainly offered by $\mathrm{TpPa}-1$ networks, the bouquet-shaped magnetic TpPa-1 owns significant enrichment capacity compared to the single core-shell structure attributing to the higher proportion of TpPa-1. Furthermore, this well-designed structure is more conducive to magnetic retrieval than monodisperse particles. To evaluate the performance of the magnetic TpPa-1 in MSPE, PAHs were chosen as the model analytes on account of their carcinogenic and genotoxic (DNA-damaging) potentials. As a result, this effective MSPE pretreatment coupled with HPLCFLD was determined to be a reliable method for analysis of trace PAHs in environmental samples, which provides a fresh idea of exploring the potential applications of COFs for environmental issues.

\section{EXPERIMENTAL SECTION}

Reagents and Chemicals. Fluoranthene (FluA), pyrene (Pyr), benzo $(a)$ anthracene $(\mathrm{BaA})$, benzo[b]fluoranthene $(\mathrm{BbF})$, benzo $(a)$ pyrene $(\mathrm{BaP})$, and benzo $[\mathrm{g}, h, i]$ perylene $(\mathrm{BghiP})$ were purchased from AccuStandard (New Haven, CT). 1,3,5-Triformylphloroglucinol, $p$ phenylenediamine and 1,6-hexanediamine were obtained from J\&K
Chemical Co. Ltd. (Beijing, China). Ferric chloride hexahydrate $\left(\mathrm{FeCl}_{3} \cdot 6 \mathrm{H}_{2} \mathrm{O}\right)$, sodium acetate (NaAc), N,N-dimethylformamide (DMF), glycol, and anhydrous ethanol were from Sinopharm Chemistry Reagent Co. Ltd. (Beijing, China). HPLC-grade acetonitrile, acetone and methanol were supplied by Fisher Scientific (Fair Lawn, NJ). Humic acid was provided by Sigma-Aldrich (St. Louis, $\mathrm{MO})$. Ultrapure water was produced by using Milli-Q SP water purification system (Millipore, Bedford, MA, U.S.A.).

Synthesis of the Gypsophila Bouquet-Shaped Magnetic COF. The amino-functionalized $\mathrm{Fe}_{3} \mathrm{O}_{4}$ nanoparticles $\left(\mathrm{NH}_{2}-\mathrm{Fe}_{3} \mathrm{O}_{4}\right.$ NPs) were synthesized by a previously reported method. ${ }^{40}$ The asprepared $\mathrm{NH}_{2}-\mathrm{Fe}_{3} \mathrm{O}_{4} \mathrm{NPs}$ were introduced to a solution of $20 \mathrm{~mL}$ of ethanol containing 1,3,5-triformylphoroglucinol (Tp) and then maintained for $1 \mathrm{~h}$ at $50{ }^{\circ} \mathrm{C}$ to obtain surface-modified magnetic nanoparticles. This process helps trigger the coverage by COF due to the Schiff base reaction, by which the monomer $\mathrm{Tp}$ was preferentially fixed on the surface of magnetic nanoparticles. ${ }^{41}$ After each step, the synthesized nanoparticles could be collected from the mixture for rinsing with the help of a magnet.

Bouquet-like magnetic COF was prepared by a facile roomtemperature solution-phase approach. ${ }^{42}$ 1,3,5-Triformylphoroglucinol $(\mathrm{Tp}, 0.15 \mathrm{mmol})$ and $p$-phenylenediamine $(\mathrm{Pa}-1,0.225 \mathrm{mmol})$ were separately dissolved with $20 \mathrm{~mL}$ of ethanol and then added to $20 \mathrm{~mL}$ of surface-modified magnetic nanoparticles under mechanical agitation at room temperature. After the solution was stirred for $30 \mathrm{~min}$, the brown colored magnetic TpPa-1 was magnetically isolated and washed with DMF to remove unreacted residual $\mathrm{Tp}$ and $\mathrm{Pa}-1$, washed with ethanol, and lastly, dried under vacuum at $50{ }^{\circ} \mathrm{C}$. For comparison, pure $\mathrm{TpPa}-1$ was synthesized via the above-mentioned method but without the addition of surface-modified $\mathrm{Fe}_{3} \mathrm{O}_{4}$ nanoparticles.

Characterization. Hitachi S-5500 field-emission scanning electron microscope (FE-SEM, Tokyo, Japan) and Hitachi H-7500 transmission electron microscope (TEM, Tokyo, Japan) were applied to characterize the morphology of the synthesized materials. Infrared absorption spectra were recorded on a Nicolet Thermo NEXUS 670 Fourier transform infrared spectrometer (FT-IR, Waltham, MA) in the wavelength range of $4000-500 \mathrm{~cm}^{-1}$. X-ray diffraction analyses (XRD, PANalytical $X^{\prime}$ Pert diffractometer, Almelo, Netherlands) were performed on a monochromatized X-ray beam with nickel-filtered copper radiation at $40 \mathrm{kV}$ and $30 \mathrm{~mA}$. Surface analysis by X-ray photoelectron spectroscopy (XPS) was carried out using a Thermo Scientific ESCA-Lab-200i-XL equipment (Waltham, MA), and the Xray source was $\mathrm{Al} \mathrm{K} \alpha$ radiation (1486.6 eV, monochromatic). Thermogravimetric analysis (TGA) was measured with TGA Q5000 IR thermogravimetric analyzer (TA Instruments, New Castle, U.S.A.) with the heating rate of $10{ }^{\circ} \mathrm{C} \mathrm{min}{ }^{-1}$ under $\mathrm{N}_{2}$ atmosphere. Elemental analysis was conducted on EA3000 analyzer (EUROVECTOR). The solid-state NMR spectrum was obtained on a Bruker AVANCE III 400 $\mathrm{MHz}$ spectrometer. Magnetization was detected with a LDJ9600 vibrating sample magnetometer (VSM, Troy, MI) at ambient 

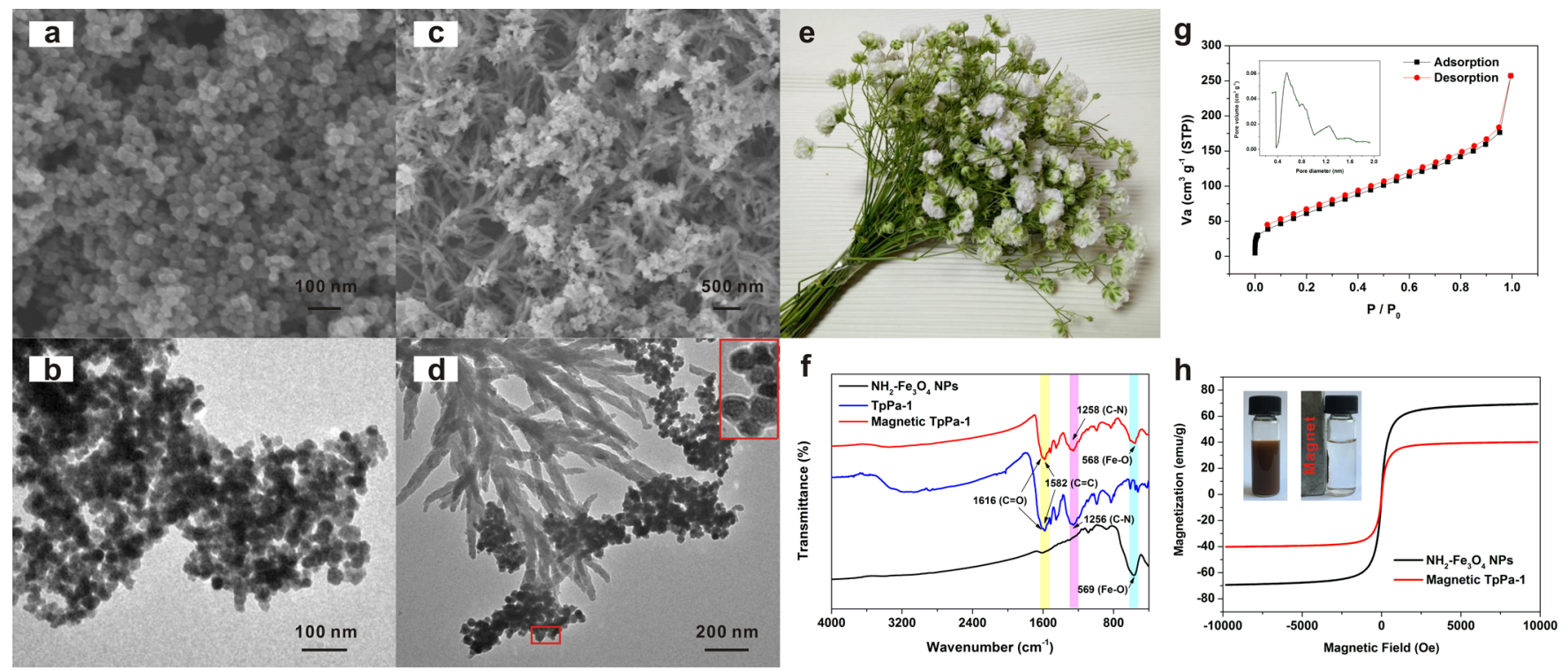

Figure 1. SEM images of the $\mathrm{NH}_{2}-\mathrm{Fe}_{3} \mathrm{O}_{4}$ (a) and magnetic TpPa-1 (c); TEM images of the $\mathrm{NH}_{2}-\mathrm{Fe}_{3} \mathrm{O}_{4}$ (b) and magnetic TpPa-1 (d); (e) photo of gypsophila bouquet; (f) FT-IR spectra of the $\mathrm{NH}_{2}-\mathrm{Fe}_{3} \mathrm{O}_{4}$, TpPa-1, and magnetic TpPa-1; (g) Nitrogen adsorption-desorption isotherm of the bouquet-shaped magnetic TpPa-1, inset: pore-size distribution of this nanocomposites; (h) magnetization hysteresis loops of the $\mathrm{NH}_{2}-\mathrm{Fe}_{3} \mathrm{O}_{4}$ and magnetic TpPa-1.

temperature. Nitrogen sorption isotherm was measured at $77 \mathrm{~K}$ using a Quadrasorb SI equipped with surface area and pore size analyzer (Quantachrome Instruments, Boynton Beach, FL).

MSPE Procedure. Five milligrams of the magnetic TpPa-1 was directly dispersed into $200 \mathrm{~mL}$ of filtered water sample spiked with the proper amount of PAHs. The mixture was first sonicated for $1 \mathrm{~min}$ and was then subjected to additional shaking for another $20 \mathrm{~min}$. After extraction of the analytes, an external magnet was utilized to gather the sorbent. The supernatant was decanted followed by addition of $12 \mathrm{~mL}$ of acetonitrile $(3 \mathrm{~mL} \times 4)$ to desorb PAHs from the isolated sorbent under ultrasound. The eluate was collected and concentrated to less than $1 \mathrm{~mL}$ with a stream of $\mathrm{N}_{2}$ at $55^{\circ} \mathrm{C}$. Then the residue was diluted to $1 \mathrm{~mL}$ with acetonitrile. Finally, $20 \mu \mathrm{L}$ of the resulting solution was injected into HPLC-FLD system.

Instrumental and Analytical Conditions. The PAHs were separated and determined using a Dionex high-performance liquid chromatography (HPLC) system equipped with a P680 pump, an ASI100 automatic sampler, a DIONEX RF 2000 fluorescence detector, a Diamonsil C18 column $(250 \times 4.6 \mathrm{~mm}, 5 \mu \mathrm{m})$, and Chromeleon 6.70 software. The mobile phase was a binary solvent mixture of acetonitrile-water (50:50, volume ratio), and the flow rate was set as $1.0 \mathrm{~mL} \mathrm{~min}{ }^{-1}$. The gradient elution program was as follows: in the initial $15 \mathrm{~min}$, maintained at $80 \%$ acetonitrile, then linear gradient to $100 \%$ acetonitrile within $1 \mathrm{~min}$, and holding for $4 \mathrm{~min}$. The excitation/ emission wavelengths pairs were as follows: 252/370 nm for fluoranthene (FluA), 280/460 nm for pyrene (Pyr), 270/390 nm for benzo(a)anthracene (BaA), 260/432 nm for benzo[b]fluoranthene (BbF), 290/410 nm for benzo(a)pyrene (BaP), 252/370 nm for benzo $[g, h, i]$ perylene (BghiP).

With a similar enrichment and analysis procedure, the effects of sorbent dosage, $\mathrm{pH}$, ionic strength, and humic acid on extraction of $\mathrm{PAHs}$ with magnetic TpPa- 1 sorbent were systematically assessed by the recoveries of the targets.

Sample Preparation. In order to study the validity of this novel sorbent, we collected three different types of water samples across Beijing. River water, lake water, and tap water samples were obtained from Shidu Scenic Spot, Olympic Forest Park, and Research Center for Eco-Environmental Sciences, respectively. All these water samples were stored at $4{ }^{\circ} \mathrm{C}$ until analysis. Before extraction, samples were filtered through $0.22 \mu \mathrm{m}$ nylon membranes.

\section{RESULTS AND DISCUSSION}

Fabrication of the Magnetic TpPa-1. Before roomtemperature synthesis of the magnetic TpPa-1, the $\mathrm{NH}_{2}-\mathrm{Fe}_{3} \mathrm{O}_{4}$ NPs were first functionalized with $\mathrm{Tp}$. The surface modification procedure for $\mathrm{NH}_{2}-\mathrm{Fe}_{3} \mathrm{O}_{4} \mathrm{NPs}$ was an indispensable preliminary step to generate binding sits or reactive centers. In this way, the local concentration of $\mathrm{Tp}$ on the $\mathrm{Fe}_{3} \mathrm{O}_{4} \mathrm{NPs}$ surface was relatively high, which induces the $\mathrm{TpPa}-1$ growth. Furthermore, the growing pattern of $\mathrm{TpPa}-1$ around magnetic nanoparticles from random tended to be directed. Figure S1 (Supporting Information) illustrates the aldehyde group of $\mathrm{Tp}$ reacted with the amino group on the surface of $\mathrm{Fe}_{3} \mathrm{O}_{4} \mathrm{NPs}$ and was successfully grafted to $\mathrm{Fe}_{3} \mathrm{O}_{4}$ NPs.

Characterization of the Hybrid Magnetic TpPa-1. The morphologies of synthesized nanomaterials were characterized by scanning electron microscopy (SEM) and transmission electron microscopy (TEM). Spherical amino-functionalized $\mathrm{Fe}_{3} \mathrm{O}_{4}$ nanoparticles $\left(\mathrm{NH}_{2}-\mathrm{Fe}_{3} \mathrm{O}_{4} \mathrm{NPs}\right)$ are approximately 30 $\mathrm{nm}$ in diameter as revealed in both SEM and TEM images in Figure 1a,b. TpPa-1 sprouted interconnected nanofibers from clustered $\mathrm{NH}_{2}-\mathrm{Fe}_{3} \mathrm{O}_{4}$ NPs (Figure 1c,d), which endows magnetic TpPa-1 with the peculiar structure similar to that of gypsophila bouquet (Figure 1e). Magnetic nanoparticles for separation and $\mathrm{TpPa}-1$ for adsorption are linked via covalent bonds. From the magnification of Figure $1 \mathrm{~d}$ marked in the red box, it can be observed that a thin layer of TpPa-1 is coated on the $\mathrm{Fe}_{3} \mathrm{O}_{4}$ NPs surface, which can be effective to protect the inner magnetic core from corrosion and oxidation. To clarify the formation mechanism for such a peculiar bouquet-like shape, the morphology characteristics of the obtained nanocomposites with different formation times were investigate by TEM analysis. Figure S2a symbolically depicts the growth pattern of the magnetic TpPa-1 with particular structure. Initially, TpPa-1 was covered on the surface of clustered $\mathrm{Fe}_{3} \mathrm{O}_{4}$ NPs, and then it began to grow thread-like structure (Figure $\mathrm{S} 2 \mathrm{~b}$ ). When the formation time reached to $30 \mathrm{~min}$, the branch- 

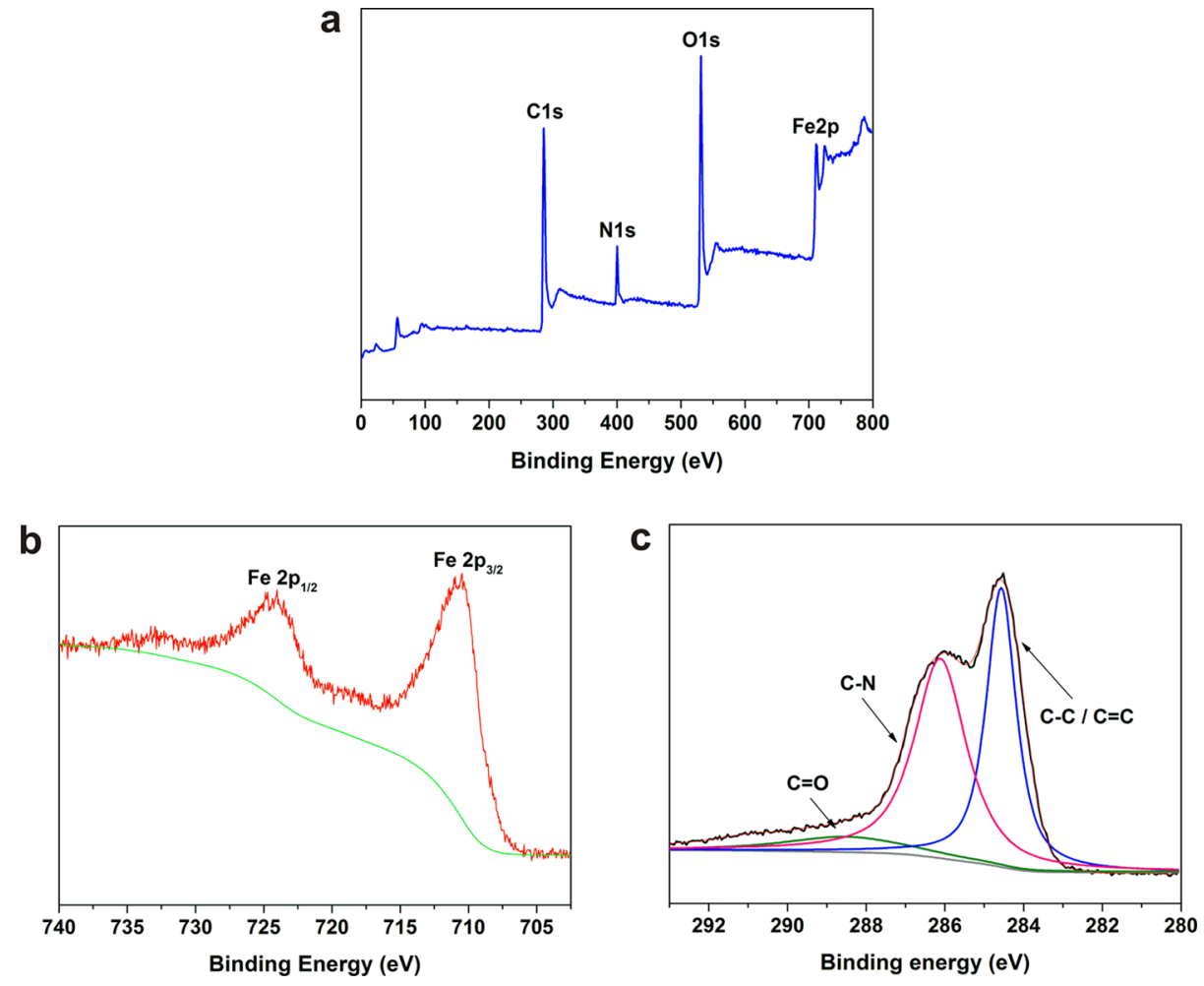

Figure 2. XPS survey spectrum (a), Fe 2p (b) and C 1s (c) spectra of magnetic TpPa-1.

type $\mathrm{TpPa}-1$ interconnected each other to develop a bouquetlike morphology (Figure S2d).

The formation of COF (TpPa-1) was first confirmed by elemental analysis, Fourier transform-infrared spectroscopy (FT-IR), and solid-state nuclear magnetic resonance (NMR) spectroscopy. The elemental analysis result manifested that the pure TpPa-1 has approximate values of $\mathrm{C}(67.74 \%), \mathrm{H}$ (4.23\%), N (12.87\%), and $\mathrm{O}(12.06 \%)$ contents to the theoretical values for an infinite $2 \mathrm{D}$ sheet (calcd for $\mathrm{C}_{18} \mathrm{H}_{12} \mathrm{~N}_{3} \mathrm{O}_{3}$ : C $67.92 \%, \mathrm{H} 3.80 \%, \mathrm{~N} 13.20 \%$, O $15.08 \%$ ). The FT-IR spectrum of TpPa-1 clearly exhibits the complete disappearance of the $\mathrm{N}-\mathrm{H}$ stretching bands (3100-3400 $\left.\mathrm{cm}^{-1}\right)$ of Pa-1 and the carbonyl stretching band $\left(1639 \mathrm{~cm}^{-1}\right)$ of Tp compared with its monomers (Figure S4). Interestingly, in the FT-IR spectrum of $\mathrm{TpPa}-1$, the $-\mathrm{OH}$ and $\mathrm{C}=\mathrm{N}$ characteristic stretching vibration peaks that should be ascribed to enol form vanished while $\mathrm{C}=\mathrm{C}$ stretching peak at 1582 $\mathrm{cm}^{-1}$ appeared, which may be attributed to the transformation from enol to keto tautomerism. ${ }^{38,39}$ The broadening peak located at $1580-1620 \mathrm{~cm}^{-1}$ could be explained by the merging of $\mathrm{C}=\mathrm{O}$ peak and $\mathrm{C}=\mathrm{C}$ stretching band. The ${ }^{13} \mathrm{C}$ crosspolarization with magic-angle spinning (CP-MAS) solid-state NMR spectrum exhibits a distinct signal near 180 ppm corresponding to the carbonyl carbon, further supporting the keto form of TpPa-1 (Figure S3). Moreover, the existence of all the characteristics peaks of TpPa-1 in the FT-IR spectrum of magnetic TpPa-1 confirms that the chemical environment within the COF remained intact (Figure 1f). The representative peaks for $\mathrm{C}=\mathrm{C}\left(1582 \mathrm{~cm}^{-1}\right)$ and $\mathrm{C}-\mathrm{N}\left(1256 \mathrm{~cm}^{-1}\right)$ stretching of $\mathrm{TpPa}-1$ remained after the integration of magnetic nanoparticles with $\mathrm{TpPa}-1$ matrix, affirming the magnetic loading could not break the basic TpPa-1 architecture. The bands at around $568 \mathrm{~cm}^{-1}$ observed in the spectra of the $\mathrm{NH}_{2}-$ $\mathrm{Fe}_{3} \mathrm{O}_{4} \mathrm{NPs}$ and magnetic TpPa-1 are attributed to the vibration of the $\mathrm{Fe}-\mathrm{O}$ bond. Furthermore, XRD pattern of the asprepared magnetic TpPa-1 with peaks for (220), (311), (400), (422), (511), and (440) planes appearing at $2 \theta=30.1^{\circ}, 35.4^{\circ}$, $43.1^{\circ}, 53.4^{\circ}, 57.5^{\circ}$, and $62.9^{\circ}$, manifests the successful loading of $\mathrm{Fe}_{3} \mathrm{O}_{4}$ NPs (according to JCPDS 19-0629) without destruction of the framework integrity and crystallinity (Figure S5).

The permanent porosity of magnetic $\mathrm{TpPa}-1$ was assessed by measuring nitrogen sorption isotherm at $77 \mathrm{~K}$. The magnetic TpPa-1 exhibits a typical type-I reversible isotherm characteristic of a microporous nature (Figure 1g). The BrunauerEmmett-Teller (BET) surface area of the bouquet-shaped magnetic TpPa-1 was estimated to be $247.8 \mathrm{~m}^{2} \mathrm{~g}^{-1}$ (compared to the value of $535 \mathrm{~m}^{2} \mathrm{~g}^{-1}$ for pure $\mathrm{TpPa}-1$ in the previous report ${ }^{38}$ ), whose decrease was caused by the addition of magnetic nanoparticles. The pore volume of the as-prepared magnetic TpPa- 1 was $0.40 \mathrm{~cm}^{3} \mathrm{~g}^{-1}$. The pore size distribution curves showed pore sizes in the range of $0.4-2.0 \mathrm{~nm}$ (inset of Figure $1 \mathrm{~g}$ ). This high ratio of surface area to total pore volume indicates the superior adsorption performance of magnetic TpPa-1. Thermogravimetric analysis (TGA) data revealed that the thermal stability of magnetic COF ( $\mathrm{TpPa}-1)$ is comparable to pure $\mathrm{TpPa}-1$, which is stable up to $300{ }^{\circ} \mathrm{C}$ (Figure S6). A slump in weight observed around $600{ }^{\circ} \mathrm{C}$ is attributed to the Curie point of magnetite.

The vibrating sample magnetization (VSM) curves of $\mathrm{NH}_{2}-$ $\mathrm{Fe}_{3} \mathrm{O}_{4} \mathrm{NPs}$ and magnetic TpPa-1 are shown in Figure 1h. The superparamagnetism nature of these materials can be proved by the hysteresis loops in the absence of apparent hysteresis, remanence, and coercivity. After cross-linking with $\mathrm{TpPa}-1$, the saturation magnetization of magnetic $\mathrm{TpPa}-1$ decreases from $69.4 \mathrm{emu} \mathrm{g}^{-1}$ to $40.1 \mathrm{emu} \mathrm{g}^{-1}$. However, it is still strong enough for magnetic isolation (as shown in the top-left inset of Figure 1h). 
To further analyze the chemical composition of the magnetic TpPa-1, X-ray photoelectron spectrometry (XPS) was carried out. In Figure $2 \mathrm{a}$, the wide scan spectra of the magnetic TpPa-1 display photoelectron lines at binding energies of 285, 402, 528, and $711 \mathrm{eV}$, which are attributed to $\mathrm{C} 1 \mathrm{~s}, \mathrm{~N} \mathrm{1s}, \mathrm{O} 1 \mathrm{~s}$, and $\mathrm{Fe}$ $2 \mathrm{p}$, respectively. Figure $2 \mathrm{~b}$ depicts the high-resolution $\mathrm{Fe} 2 \mathrm{p}$ XPS spectrum. Double characteristic binding energy peaks at 710.9 and $725.1 \mathrm{eV}$ correspond to $\mathrm{Fe} 2 \mathrm{p}_{3 / 2}$ and $\mathrm{Fe} 2 \mathrm{p}_{1 / 2}$, indicating the existence of magnetite in the composite. The $\mathrm{C}$ 1s XPS spectrum of magnetic TpPa-1 can be deconvoluted into three individual component peaks located at 284.5, 285.6, and $288.5 \mathrm{eV}$ (Figure 2c), which are assigned to $\mathrm{C}-\mathrm{C} / \mathrm{C}=\mathrm{C}, \mathrm{C}-$ $\mathrm{N}$, and $\mathrm{C}=\mathrm{O}$, respectively. These results confirm the successful formation of magnetic TpPa-1 nanocomposite with covalent bonding linkage.

Extraction Characteristics of $\mathrm{TpPa}-1$ to Organic Targets. According to the structure of TpPa-1 (Scheme 1), it is known that $\mathrm{TpPa}-1$ possesses large $\pi-\pi$ framework, as well as high percentage of $\mathrm{N}$ and $\mathrm{O}$ atoms. In consideration of this unique structure, it can be anticipated that $\mathrm{TpPa}-1$ may extract organic targets containing a benzene ring, amino or hydroxyl groups. Experiments were performed to verify the extraction validity of TpPa-1 to PAHs. The results revealed that TpPa-1 extracted these organic targets effectively. It was believed that the main impetus of TpPa-1 for extraction is dictated by hydrophobic interaction, with the assistance of hydrogen bonding, $\pi-\pi$ stacking interaction, and highly porous property. PAHs are a type of persistent organic pollutant, which are potent toxicant, carcinogens, and mutagens and may be finally transferred to humans. Six PAHs were selected as targets for evaluation of the extraction performance of the hybrid magnetic TpPa-1.

Optimization of the MSPE Conditions. A high-performance liquid chromatography with fluorescence detection (HPLC-FLD) system was applied to determine PAHs after enrichment with magnetic TpPa-1 sorbent from $200 \mathrm{~mL}$ of distilled water spiked with $50 \mathrm{ng} \mathrm{L}^{-1}$ of PAHs (as depicted in Scheme 1). Potential influences in terms of sorbent dosage, solution $\mathrm{pH}$, salt concentration, as well as macromolecular distractors were investigated and optimized on the basis of the recoveries of PAHs.

The increase of sorbent dosage is beneficial for the enhancement of surface area and accessible sites to the adsorption of the analytes; however, excessive sorbent is unprofitable. In order to achieve high extraction efficiency for PAHs, different amounts of the sorbent were examined in the range of 1-20 mg, and the results are presented in Figure S7. As shown, $5 \mathrm{mg}$ of the magnetic TpPa-1 was enough for quantitative extraction of all the six analytes, and the recoveries of most PAHs were up to $90 \%$. Also, the sample volume is an important parameter influencing the preconcentration factor and the limit of detection. Importantly, 100-1000 $\mathrm{mL}$ of spiked water with the constant amount of each PAHs (10 ng) were preconcentrated under the optimum conditions. Figure S8 shows the recoveries of PAHs from the various volumes of sample solutions. It is found that the maximum sample volume was up to $200 \mathrm{~mL}$ with the recoveries above $90 \%$, and even when the sample volume reached $1000 \mathrm{~mL}$, the recoveries of all the PAHs still retained more than $70 \%$. Compared with other reported sorbents, the ratio of the extraction solution volume $(200 \mathrm{~mL})$ to the amount of this novel sorbent $(5 \mathrm{mg})$ is higher (summarized in Table S1), which indicated a higher extraction efficiency and better enhancement ability of magnetic TpPa-1.
The possible reasons might be the large specific surface area, high porosity as well as low density of $\mathrm{TpPa}-1$, and the unique bouquet-like structure of this magnetic nanocomposite.

The influence of $\mathrm{pH}$ on extraction efficiency was shown in Figure S9. The recoveries of the target analytes remain nearly constant over the whole studied $\mathrm{pH}$ range from 3 to 11 , which may be originated from the hydrophobic effect and $\pi-\pi$ stacking interaction of $\mathrm{TpPa}-1$ toward the nonpolar PAHs molecules, and this has no apparent connection to solution $\mathrm{pH}$. In this regard, a tedious adjustment of solution $\mathrm{pH}$ can be omitted before MSPE. Additionally, the potential effect of ionic strength on the MSPE was systematically investigated by addition of a certain amount of $\mathrm{NaCl}$, and the result revealed a negligible change in adsorption performance (Figure S10). The recoveries of most PAHs could approach $90 \%$ despite that the salinity was as high as $0.1 \mathrm{~mol} \mathrm{~L}^{-1}$. The results illustrated that the as-synthesized magnetic nanoporous material is $\mathrm{pH}$ independent and well tolerated salt, thus greatly enlarging the prospect of practice.

Humic acid is widespread in environmental samples and severely impacts extraction efficiency. Using humic acid as a model macromolecule, the anti-interference capacity of magnetic TpPa-1 in MSPE was further evaluated. Due to the coverage of active sites by excess humic acid on the sorbent surface and the competitive adsorption toward PAHs, it is hardly unexpected that recoveries of targets would be reduced if some humic acid was added in the sample. This was confirmed by the results of Figure S11, in which the recoveries of all targets were markedly reduced as the concentration of humic acid increased above $40 \mathrm{mg} \mathrm{L}^{-1}$. However, the content of humic acid in natural water is always below $5 \mathrm{mg} \mathrm{L}^{-1}$, and the recoveries of PAHs were higher than $70 \%$ in this concentration range, which can satisfy requirements in environmental analysis of trace components. Such an anti-interference ability of the asprepared materials in low concentration of humic acid stems from the molecular sieving effect of the microporous TpPa-1 shell to block large molecules.

Analytical Performance and Application. Quantitative parameters such as linear range, correlation coefficient, and limit of detection of the established method under the aboveoptimized conditions were displayed in Table S2. Satisfactory linearity for the analytes was obtained in the concentration range of $2.0-200.0 \mathrm{ng} \mathrm{L}^{-1}$ with the correlation coefficients $\left(R^{2}\right)$ above 0.9993 . The limits of detection (LODs), calculated on the basis of signal-to-noise ratio of 3 , are in the range from 0.24 to $1.01 \mathrm{ng} \mathrm{L}^{-1}$. Furthermore, the established method based on magnetic TpPa-1 was successfully employed to analyze the PAHs concentration in three environmental water samples, including tap water, lake water, and river water (Table S3). From the results, it can be found all the recoveries of PAHs in these samples are in the range of $73-110 \%$ with a low relative standard deviation $(2-8 \%)$. These adequately demonstrate the applicability and reliability of this approach in real sample analysis.

\section{CONCLUSIONS}

In summary, a magnetic bouquet-shaped COF ( $\mathrm{TpPa}-1)$ has been successfully fabricated by a simple and facile roomtemperature solution-phase approach, and it was employed as a sorbent for MSPE in environmental samples. The as-prepared nanocomposite has a large surface area, supermagnetism, high water-stability, and excellent extraction performance. Additionally, because of its structural distinctiveness, the magnetic 
TpPa-1 is easier for magnetic retrieval and contains a higher proportion of COF compared to a single core-shell structure. As expected, the established MSPE procedure coupled with HPLC-FLD detection was proved to be effective for analyzing PAHs in environmental samples with satisfactory precision and accuracy. Encouraged by the effective extraction of PAHs with this magnetic three-dimensional bouquet-like covalent organic framework, it can be expected that this material may also have strong affinity to other chemicals. Future work will be directed toward further application of this material in wider fields, as well as the study of different kinds of magnetic COFs and their morphology or modification on the effect of adsorption capability.

\section{ASSOCIATED CONTENT}

\section{S Supporting Information}

The Supporting Information is available free of charge on the ACS Publications website at DOI: 10.1021/acsami.6b13643.

$$
\text { Additional figures and tables (PDF) }
$$

\section{AUTHOR INFORMATION}

\section{Corresponding Author}

*E-mail: caiyaqi@rcees.ac.cn. Fax: +86-10-6284-9182. Tel.: +86-10-6284-9239.

\section{ORCID ${ }^{\circ}$}

Sijing He: 0000-0001-9279-9267

\section{Notes}

The authors declare no competing financial interest.

\section{ACKNOWLEDGMENTS}

We thank the National Key Research and Development Program (2016YFA0203102), the National Natural Science Foundation of China (21537004, 21477140, 21321004, 21277152), and Strategic Priority Research Program of the Chinese Academy of Sciences (XDB14010201) for financial support.

\section{REFERENCES}

(1) Chen, L.; Wang, T.; Tong, J. Application of Derivatized Magnetic Materials to the Separation and the Preconcentration of Pollutants in Water Samples. TrAC, Trends Anal. Chem. 2011, 30, 1095-1108.

(2) Mahmoud, M. E.; Ahmed, S. B.; Osman, M. M.; Abdel-Fattah, T. M. A Novel Composite of Nanomagnetite-Immobilized-Baker's Yeast on the Surface of Activated Carbon for Magnetic Solid Phase Extraction of $\mathrm{Hg}(\mathrm{II})$. Fuel 2015, 139, 614-621.

(3) Rodríguez, J. A.; Escamilla-Lara, K. A.; Guevara-Lara, A.; Miranda, J. M.; Páez-Hernández, M. E. Application of an Activated Carbon-Based Support for Magnetic Solid Phase Extraction Followed by Spectrophotometric Determination of Tartrazine in Commercial Beverages. Int. J. Anal. Chem. 2015, 2015, 291827.

(4) Zhang, S.; Yao, W.; Ying, J.; Zhao, H. Polydopamine-Reinforced Magnetization of Zeolitic Imidazolate Framework ZIF-7 for Magnetic Solid-Phase Extraction of Polycyclic Aromatic Hydrocarbons from the Air-Water Environment. J. Chromatogr. A 2016, 1452, 18-26.

(5) Liu, X.; Sun, Z.; Chen, G.; Zhang, W.; Cai, Y.; Kong, R.; Wang, X.; Suo, Y.; You, J. Determination of Phthalate Esters in Environmental Water by Magnetic Zeolitic Imidazolate Framework-8 SolidPhase Extraction Coupled with High-Performance Liquid Chromatography. J. Chromatogr. A 2015, 1409, 46-52.

(6) Zhou, L.; Hu, Y.; Li, G. Conjugated Microporous Polymers with Built-In Magnetic Nanoparticles for Excellent Enrichment of Trace Hydroxylated Polycyclic Aromatic Hydrocarbons in Human Urine. Anal. Chem. 2016, 88, 6930-6938.
(7) Hu, Y.; Huang, Z.; Liao, J.; Li, G. Chemical Bonding Approach for Fabrication of Hybrid Magnetic Metal-Organic Framework-5: High Efficient Adsorbents for Magnetic Enrichment of Trace Analytes. Anal. Chem. 2013, 85, 6885-6893.

(8) Huo, S.; Yan, X. Facile Magnetization of Metal-Organic Framework MIL-101 for Magnetic Solid-Phase Extraction of Polycyclic Aromatic Hydrocarbons in Environmental Water Samples. Analyst 2012, 137, 3445-3451.

(9) Chen, X.; Ding, N.; Zang, H.; Yeung, H.; Zhao, R.; Cheng, C.; Liu, J.; Chan, T.-W. D. $\mathrm{Fe}_{3} \mathrm{O}_{4} @ \mathrm{MOF}$ Core-Shell Magnetic Microspheres for Magnetic Solid-Phase Extraction of Polychlorinated Biphenyls from Environmental Water Samples. J. Chromatogr. A 2013, 1304, 241-245.

(10) Zhang, W.; Yan, Z.; Gao, J.; Tong, P.; Liu, W.; Zhang, L. MetalOrganic Framework UiO-66 Modified Magnetite@Silica Core-Shell Magnetic Microspheres for Magnetic Solid-Phase Extraction of Domoic Acid from Shellfish Samples. J. Chromatogr. A 2015, 1400, $10-18$.

(11) Wang, Y.; Xie, J.; Wu, Y.; Ge, H.; Hu, X. Preparation of a Functionalized Magnetic Metal-Organic Framework Sorbent for the Extraction of Lead Prior to Electrothermal Atomic Absorption Spectrometer Analysis. J. Mater. Chem. A 2013, 1, 8782-8789.

(12) Yang, Y.; Ma, X.; Feng, F.; Dang, X.; Huang, J.; Chen, H. Magnetic Solid-Phase Extraction of Triclosan Using Core-Shell $\mathrm{Fe}_{3} \mathrm{O}_{4} @$ MIL-100 Magnetic Nanoparticles, and Its Determination by HPLC with UV Detection. Microchim. Acta 2016, 183, 2467-2472.

(13) Zhao, X.; Cai, Y.; Wang, T.; Shi, Y.; Jiang, G. Preparation of Alkanethiolate-Functionalized Core/Shell $\mathrm{Fe}_{3} \mathrm{O}_{4} @ A u$ Nanoparticles and Its Interaction with Several Typical Target Molecules. Anal. Chem. 2008, 80, 9091-9096.

(14) Ding, S.; Wang, W. Covalent Organic Frameworks (COFs): from Design to Applications. Chem. Soc. Rev. 2013, 42, 548-568.

(15) Feng, X.; Ding, X.; Jiang, D. Covalent Organic Frameworks. Chem. Soc. Rev. 2012, 41, 6010-6022.

(16) Ockwig, N. W.; Delgado-Friedrichs, O.; O’Keeffe, M.; Yaghi, O. M. Reticular Chemistry: Occurrence and Taxonomy of Nets and Grammar for the Design of Frameworks. Acc. Chem. Res. 2005, 38, 176-182.

(17) Furukawa, H.; Yaghi, O. M. Storage of Hydrogen, Methane, and Carbon Dioxide in Highly Porous Covalent Organic Frameworks for Clean Energy Applications. J. Am. Chem. Soc. 2009, 131, 8875-8883.

(18) Huang, N.; Chen, X.; Krishna, R.; Jiang, D. Two-Dimensional Covalent Organic Frameworks for Carbon Dioxide Capture through Channel-Wall Functionalization. Angew. Chem., Int. Ed. 2015, 54, 2986-2990.

(19) Stegbauer, L.; Hahn, M. W.; Jentys, A.; Savasci, G.; Ochsenfeld, C.; Lercher, J. A.; Lotsch, B. V. Tunable Water and $\mathrm{CO}_{2}$ Sorption Properties in Isostructural Azine-Based Covalent Organic Frameworks through Polarity Engineering. Chem. Mater. 2015, 27, 7874-7881.

(20) Zeng, Y.; Zou, R.; Zhao, Y. Covalent Organic Frameworks for CO2 Capture. Adv. Mater. 2016, 28, 2855-2873.

(21) Ding, S.; Gao, J.; Wang, Q.; Zhang, Y.; Song, W.; Su, C.; Wang, W. Construction of Covalent Organic Framework for Catalysis: Pd/ COF-LZU1 in Suzuki-Miyaura Coupling Reaction. J. Am. Chem. Soc. 2011, 133, 19816-19822.

(22) Xu, H.; Gao, J.; Jiang, D. Stable, Crystalline, Porous, Covalent Organic Frameworks as a Platform for Chiral Organocatalysts. Nat. Chem. 2015, 7, 905-912.

(23) Fang, Q.; Gu, S.; Zheng, J.; Zhuang, Z.; Qiu, S.; Yan, Y. 3D Microporous Base-Functionalized Covalent Organic Frameworks for Size-Selective Catalysis. Angew. Chem., Int. Ed. 2014, 53, 2878-2882.

(24) Xu, H.; Chen, X.; Gao, J.; Lin, J.; Addicoat, M.; Irle, S.; Jiang, D. Catalytic Covalent Organic Frameworks via Pore Surface Engineering. Chem. Commun. 2014, 50, 1292-1294.

(25) Wu, Y.; Xu, H.; Chen, X.; Gao, J.; Jiang, D. A $\pi$-Electronic Covalent Organic Framework Catalyst: $\pi$-Walls as Catalytic Beds for Diels-Alder Reactions Under Ambient Conditions. Chem. Commun. 2015, 51, 10096-10098. 
(26) Wan, S.; Guo, J.; Kim, J.; Ihee, H.; Jiang, D. A Belt-Shaped, Blue Luminescent, and Semiconducting Covalent Organic Framework. Angew. Chem., Int. Ed. 2008, 47, 8826-8830.

(27) Dogru, M.; Handloser, M.; Auras, F.; Kunz, T.; Medina, D.; Hartschuh, A.; Knochel, P.; Bein, T. A Photoconductive Thienothiophene-Based Covalent Organic Framework Showing Charge Transfer Towards Included Fullerene. Angew. Chem., Int. Ed. 2013, 52, 29202924.

(28) Dogru, M.; Bein, T. On the Road Towards Electroactive Covalent Organic Frameworks. Chem. Commun. 2014, 50, 5531-5546.

(29) Medina, D. D.; Werner, V.; Auras, F.; Tautz, R.; Dogru, M.; Schuster, J.; Linke, S.; Döblinger, M.; Feldmann, J.; Knochel, P.; Bein, T. Oriented Thin Films of a Benzodithiophene Covalent Organic Framework. ACS Nano 2014, 8, 4042-4052.

(30) Fei, H. H.; Cahill, J. F.; Prather, K. A.; Cohen, S. M. Tandem Postsynthetic Metal Ion and Ligand Exchange in Zeolitic Imidazolate Frameworks. Inorg. Chem. 2013, 52, 4011-4016.

(31) Keskin, S.; van Heest, T. M.; Sholl, D. S. Can Metal-Organic Framework Materials Play a Useful Role in Large-Scale Carbon Dioxide Separations? ChemSusChem 2010, 3, 879-891.

(32) Gopalakrishnan, D.; Dichtel, W. R. Direct Detection of RDX Vapor Using a Conjugated Polymer Network. J. Am. Chem. Soc. 2013, 135, 8357-8362.

(33) Jiang, J.; Su, F.; Trewin, A.; Wood, C. D.; Campbell, N. L.; Niu, H.; Dickinson, C.; Ganin, A. Y.; Rosseinsky, M. J.; Khimyak, Y. Z.; Cooper, A. I. Conjugated Microporous Poly(aryleneethynylene) Networks. Angew. Chem., Int. Ed. 2007, 46, 8574-8578.

(34) Biswal, B. P.; Chandra, S.; Kandambeth, S.; Lukose, B.; Heine, T.; Banerjee, R. Mechanochemical Synthesis of Chemically Stable Isoreticular Covalent Organic Frameworks. J. Am. Chem. Soc. 2013, 135, 5328-5331.

(35) Pachfule, P.; Panda, M. K.; Kandambeth, S.; Shivaprasad, S. M.; Díaz, D. D.; Banerjee, R. Multifunctional and Robust Covalent Organic Framework-Nanoparticle Hybrids. J. Mater. Chem. A 2014, 2, 7944-7952.

(36) Biswal, B. P.; Kandambeth, S.; Chandra, S.; Shinde, D. B.; Bera, S.; Karak, S.; Garai, B.; Kharul, U. K.; Banerjee, R. Pore Surface Engineering in Porous, Chemically Stable Covalent Organic Frameworks for Water Adsorption. J. Mater. Chem. A 2015, 3, 23664-23669.

(37) Pachfule, P.; Kandambeth, S.; Díaz, D. D.; Banerjee, R. Highly Stable Covalent Organic Framework-Au Nanoparticles Hybrids for Enhanced Activity for Nitrophenol Reduction. Chem. Commun. 2014, 50, 3169-3172.

(38) Chandra, S.; Kandambeth, S.; Biswal, B. P.; Lukose, B.; Kunjir, S. M.; Chaudhary, M.; Babarao, R.; Heine, T.; Banerjee, R. Chemically Stable Multilayered Covalent Organic Nanosheets from Covalent Organic Frameworks via Mechanical Delamination. J. Am. Chem. Soc. 2013, 135, 17853-17861.

(39) Kandambeth, S.; Mallick, A.; Lukose, B.; Mane, M. V.; Heine, T.; Banerjee, R. Construction of Crystalline 2D Covalent Organic Frameworks with Remarkable Chemical (Acid/Base) Stability via a Combined Reversible and Irreversible Route. J. Am. Chem. Soc. 2012, 134, 19524-19527.

(40) Wang, L.; Bao, J.; Wang, L.; Zhang, F.; Li, Y. One-Pot Synthesis and Bioapplication of Amine-Functionalized Magnetite Nanoparticles and Hollow Nanospheres. Chem. - Eur. J. 2006, 12, 6341-6347.

(41) Tan, J.; Namuangruk, S.; Kong, W.; Kungwan, K.; Guo, J.; Wang, C. Manipulation of Amorphous-to-Crystalline Transformation: Towards the Construction of Covalent Organic Framework Hybrid Microspheres with NIR Photothermal Conversion Ability. Angew. Chem., Int. Ed. 2016, 55, 13979-13984.

(42) Yang, C.; Liu, C.; Cao, Y.; Yan, X. Facile Room-Temperature Solution-Phase Synthesis of a Spherical Covalent Organic Framework for High-Resolution Chromatographic Separation. Chem. Commun. 2015, 51, 12254-12257. 